\section{OPEN ACCESS}

Edited by:

Dora ll'yasova,

Duke University, United States

Reviewed by:

Shengxiu Liu,

First Affiliated Hospital of Anhui Medical University, China Siming Yuan,

Medical School of Nanjing University, China

*Correspondence:

Andrea Diociaiuti andrea.diociaiuti@opbg.net

Specialty section:

This article was submitted to

Children and Health,

a section of the journal

Frontiers in Pediatrics

Received: 24 June 2021 Accepted: 02 September 2021 Published: 07 October 2021

Citation:

Diociaiuti A, Paolantonio G, Zama M, Alaggio R, Carnevale C, Conforti $A$, Cesario C, Dentici ML, Buonuomo PS, Rollo $M$ and El Hachem M (2021) Vascular Birthmarks as a Clue for Complex and Syndromic Vascular

Anomalies. Front. Pediatr. 9:730393. doi: 10.3389/fped.2021.730393

\title{
Vascular Birthmarks as a Clue for Complex and Syndromic Vascular Anomalies
}

\begin{abstract}
Andrea Diociaiuti ${ }^{1 *}$, Guglielmo Paolantonio ${ }^{2}$, Mario Zama ${ }^{3}$, Rita Alaggio ${ }^{4}$, Claudia Carnevale ${ }^{1}$, Andrea Conforti ${ }^{5}$, Claudia Cesario ${ }^{6}$, Maria Lisa Dentici ${ }^{7}$, Paola Sabrina Buonuomo ${ }^{8}$, Massimo Rollo ${ }^{2}$ and May El Hachem ${ }^{1}$
\end{abstract}

\begin{abstract}
'Dermatology Unit and Genodermatosis Unit, Genetics and Rare Diseases Research Division, Bambino Gesù Children's Hospital, IRCCS, Rome, Italy, ${ }^{2}$ Interventional Radiology Unit, Department of Imaging, Bambino Gesù Children's Hospital, IRCCS, Rome, Italy, ${ }^{3}$ Craniofacial Centre-Plastic and Maxillofacial Surgery Unit, Bambino Gesù Children's Hospital, IRCCS, Rome, Italy, ${ }^{4}$ Department of Pathology, Bambino Gesù Children's Hospital, IRCCS, Rome, Italy, ${ }^{5}$ Department of Neonatal Medicine and Surgery, Bambino Gesù Children's Hospital, IRCCS, Rome, Italy, ${ }^{6}$ Laboratory of Medical Genetics, Department of Laboratories, Bambino Gesù Children's Hospital, IRCCS, Rome, Italy, ${ }^{7}$ Medical Genetics Unit, Bambino Gesù Children's Hospital, IRCCS, Rome, Italy, ${ }^{8}$ Rare Diseases and Medical Genetics Unit, Bambino Gesù Children's Hospital, IRCCS, Rome, Italy
\end{abstract}

Vascular birthmarks are common in neonates (prevalence: 20-30\%) and mostly incidental findings sometimes with spontaneous regression (salmon patch and nevus simplex). Capillary malformations are found in about $1 \%$ and infantile hemangiomas are found in $4 \%$ of mature newborns. Vascular malformations are classified according to their most prominent vessel type. The term "capillary malformation" (port wine stain) includes a wide range of vascular lesions with different characteristics; they may be isolated or part of specific syndromic conditions. Part of the infantile hemangiomas and of the vascular malformations may require treatment for functional or cosmetic reasons, and in rare cases, investigations are also necessary as they represent a clue for the diagnosis of complex vascular malformation or tumors associated with extracutaneous abnormalities. Complex vascular malformations are mostly mosaicism due to early somatic mutations. Genetic advances have led to identify the main pathogenic pathways involved in this disease group. Diffuse capillary malformation with overgrowth, Klippel-Trenaunay syndrome, CLAPO syndrome, CLOVES syndrome, and megalencephaly-capillary malformation belong to the PIK3CA-related overgrowth. Capillary malformation-arteriovenous malformation underlies a fast-flow vascular malformation, sometimes manifesting as Parkes-Weber syndrome. Recognition of these different types of capillary vascular stains is sometimes difficult; however, associated findings may orient the clinicians while genetic testing may confirm the diagnosis. Lymphatic malformation frequently manifests as large masses that compress and/or infiltrate the surrounding tissues, representing a neonatal emergency when airways are involved. Infantile hemangiomas may cause functional and/or permanent esthetical damage, depending on their localization (such as periorbital area, lip, nose); large (more than $5 \mathrm{~cm}$ ) infantile hemangiomas with a segmental distribution can be associated with obstruction or malformations of the underneath organs with complications: PHACE syndrome, 
LUMBAR/SACRAL syndrome, and beard infantile hemangioma. In our review, we discuss controversies regarding the international classification and emerging concepts in the field of vascular anomalies. Finally, we discuss potential developments of new, non-invasive diagnostic techniques and repurposing of target therapies from oncology. Complex and/or life-threatening vascular tumors and malformations are extremely rare events and they represent a considerable therapeutic challenge. Early recognition of clinical signs suggestive for a specific disease may improve therapeutic outcomes and avoid severe complications.

Keywords: vascular birthmarks, vascular anomalies, vascular tumors, vascular malformations, complex vascular malformations

\section{INTRODUCTION}

Vascular anomalies comprise a wide spectrum of disorders ranging from benign transient manifestations to complex and life-threatening diseases. The classification by the International Society for the Study of Vascular Anomalies (ISSVA) distinguishes vascular tumors from malformations (1). Tumors are subdivided into benign, locally aggressive, and malignant. Vascular malformations are defects in vasculogenesis, while vascular malformations are classified based on the basis of the most prominent vessel type (capillary, lymphatic, and venous). They also may be combined if composed by multiple vessel subtypes and may be associated with others anomalies.

ISSVA classification integrating genetic recent advances together with clinical phenotypes led to correctly identify clinical entities and avoid use of improper terms and consequent inadequate management. However, emerging diseases are not yet included in the current classification and there are some criticism about the use of some terms, such as "capillary malformation." In addition, this classification is not yet adopted for liver and cerebral vascular anomalies.

Vascular birthmarks are common in neonates with a prevalence $>30 \%$ (2) and may be undervalued by physicians. Indeed, even if the vast majority are transient or benign cutaneous manifestations (salmon patch, nevus simplex, and stork bite), in some cases, they may represent an important clue for the diagnosis of complex and syndromic vascular anomalies: vascular malformations and vascular tumors associated with extracutaneous anomalies.

Capillary malformations (CMs) are simple slow-flow vascular malformation. However, when localized on the forehead, they may be part of the Sturge-Weber syndrome (SWS). The association of overgrowth with CM belongs to the PIK3CArelated overgrowth syndromes (PROS). In addition, CMs have been described in capillary malformation-arteriovenous malformation (CM-AVM), although in this case, they are not slow-flow vascular malformations.

Cutaneous venous malformations (VMs) are slow-flow VMs. They are frequently single lesions but may be multifocal or associated with extracutaneous localizations, most frequently on the gastrointestinal tract.

Lymphatic malformations are slow-flow malformations of lymphatic vessels. They may be present at birth as voluminous masses in particular located on the head and neck, or manifest later.

Infantile hemangiomas (IHs) are the most common benign tumors in infancy with a prevalence of $4-5 \%$ (3). They usually present within the first weeks and are characterized by rapid growth in the first 4-9 months generally followed by spontaneous involution within 4-7 years, with or without scars. However, investigations and early treatment are needed in $12 \%$ of cases due to functional or permanent esthetical damage. In addition, large (more than $5 \mathrm{~cm}$ ) IH may be associated with extracutaneous malformations. This is the case of PHACE and PELVIS/LUMBAR/SACRAL syndrome.

Although complex and/or life-threatening vascular tumors and malformations are rare diseases, they usually present at birth or in the first weeks of life. Therefore, even minimal skin signs suggestive for these conditions should be taken into account in order to achieve the correct diagnosis, to prevent future complications, and to improve the quality of life of the patients and their families.

\section{MATERIALS AND METHODS}

Review of the literature of the last 15 years has been made on PubMed using the following terms: "capillary malformation," "PROS," "Sturge Weber," "Klippel-Trenaunay," “CLAPO," "CLOVES," "megalencephaly-capillary malformation," "lymphatic malformations," "infantile hemangioma," "PHACE," "PELVIS," "SACRAL," "LUMBAR," "venous malformations," "blue rubber bleb nevus syndrome", and "KasabachMerritt phenomenon." Results have been checked by two independent authors in order to exclude duplicate papers and to select the ones to read in extenso. Every author contributed to write the parts of the manuscript related to her/his specialty.

\section{RESULTS}

Since vascular birthmarks are very common and mostly benign, they may be overlooked by the neonatologist. In rare cases, these lesions are a sign of complex vascular disorders. Moreover, vascular lesions are not always present or fully developed at birth and they may manifest 

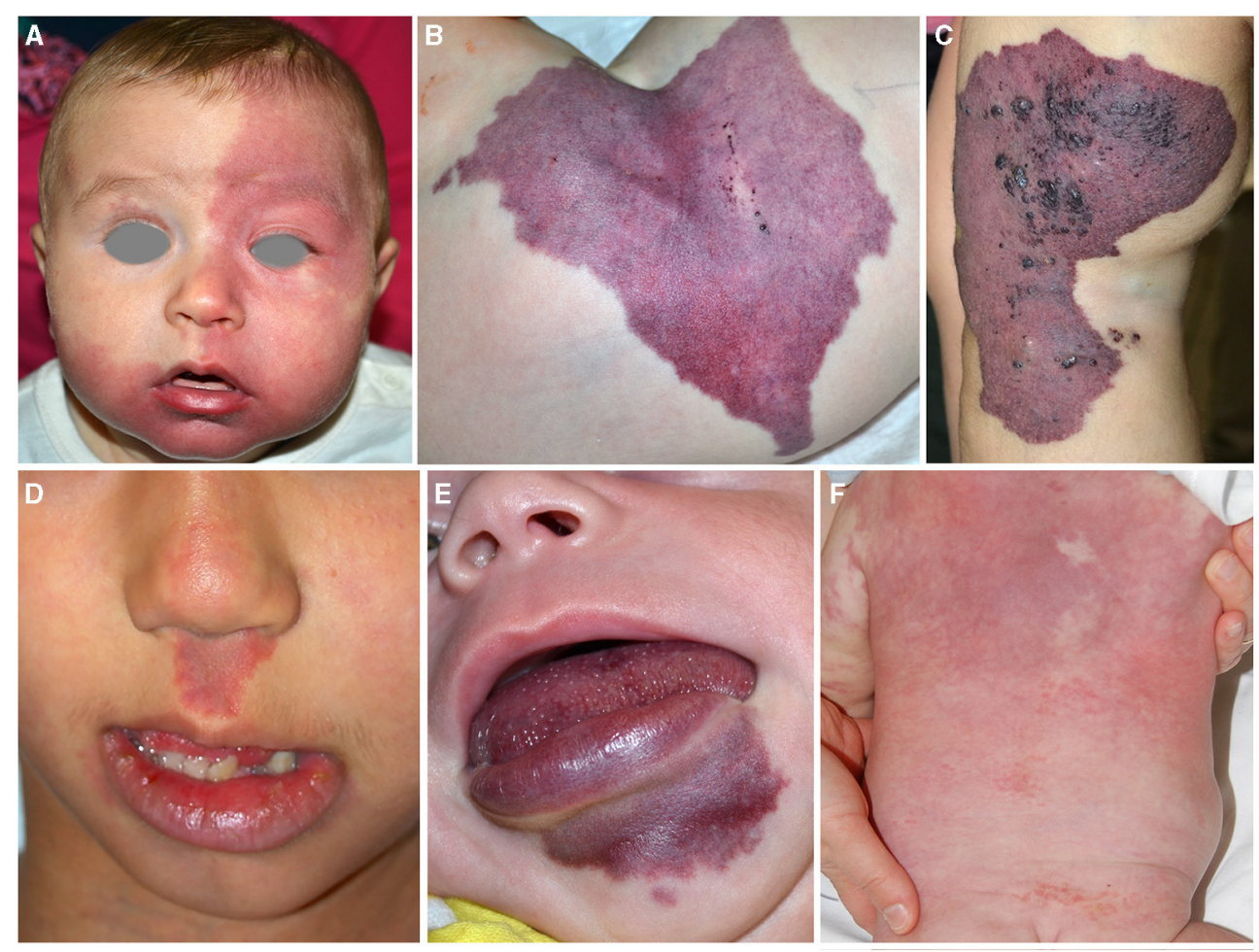

FIGURE 1 | Capillary malformation involving the frontal area in an infant affected with Sturge Weber syndrome (A). Violaceous patch of the hip in an infant affected with CLOVES syndrome. Crusted vesicles are present on the surface of the stain indicating the presence of a lymphatic component (B). Capillary-venous-lymphatic malformation of the thigh in a child affected with Klippel Trenaunay syndrome (C). Capillary malformation of the philtrum in a child affected with

megalencephaly-capillary malformation (D). Capillary malformation of the lower lip in an infant affected with CLAPO syndrome (E). Reticulated and diffuse capillary malformation with blurred margins on the trunk in a 3-month-old infant affected with diffuse capillary malformation with overgrowth (F).

during the first years of life. Thus, follow-up is essential to guarantee the correct diagnosis and to avoid misdiagnosis of complex diseases.

\section{Capillary Malformations}

CMs, the most frequent vascular malformation, are slow-flow vascular lesions constituted by dilated capillaries, arterioles, and post-capillary venules. They are frequently simple and isolated. However, CMs may be part of syndromic disorders.

Sturge-Weber syndrome (SWS). Cutaneous CMs of the face associated with leptomeningeal and/or choroidal involvement define SWS. In particular, CM localized in the forehead, delineated at its inferior border by a line joining the outer canthus of the eye to the top of the ear, and including the upper eyelid, may predict central nervous system (CNS) and/or ocular involvement (4) (Figure 1A). This association may be the consequence of the common origin from the prosencephalon of the frontonasal prominence, meninx, and encephalon. The forehead, nose, philtrum, and primary palate derives from the frontonasal prominence (5). Indeed, an early mutation in GNAQ gene in the prosencephalon may cause a complex vascular anomaly distributed in the skin, eye, and the CNS. Recently, two cases of SWS due to GNA11 have been described $(6,7)$. A somatic GNAQ mutation is present in SWS (8-10).

PIK3CA related overgrowth spectrum. CMs may be associated with segmental overgrowth and sometimes with skeletal anomalies. Somatic mutations in the phosphatidylinositol4,5-bisphosphate 3-kinase, catalytic subunit alpha (PIK3CA) gene have been identified in patients affected with various syndromes characterized by vascular anomalies and segmental overgrowth. Initially, the term PROS included Fibroadipose hyperplasia or overgrowth, hemihyperplasia multiple lipomatosis, congenital lipomatous overgrowth, vascular malformations, epidermal nevi, scoliosis/skeletal and spinal syndrome, macrodactyly, fibroadipose infiltrating lipomatosis (CLOVES), and megalencephaly-capillary malformation (MCAP) (11). Subsequently, other entities have been added to this spectrum. Indeed, PIK3CA mutations have been described in 21 patients with Klippel-Trenaunay syndrome (KTS) (12), in capillary malformation of the lower lip, lymphatic malformations of the face and neck, asymmetry, and partial or generalized overgrowth (CLAPO) syndrome (13), and in diffuse capillary malformation with overgrowth (DCMO) (14).

CM in PROS has peculiar characteristics: the color is generally violaceous with geographic borders (Figure 1B). The 

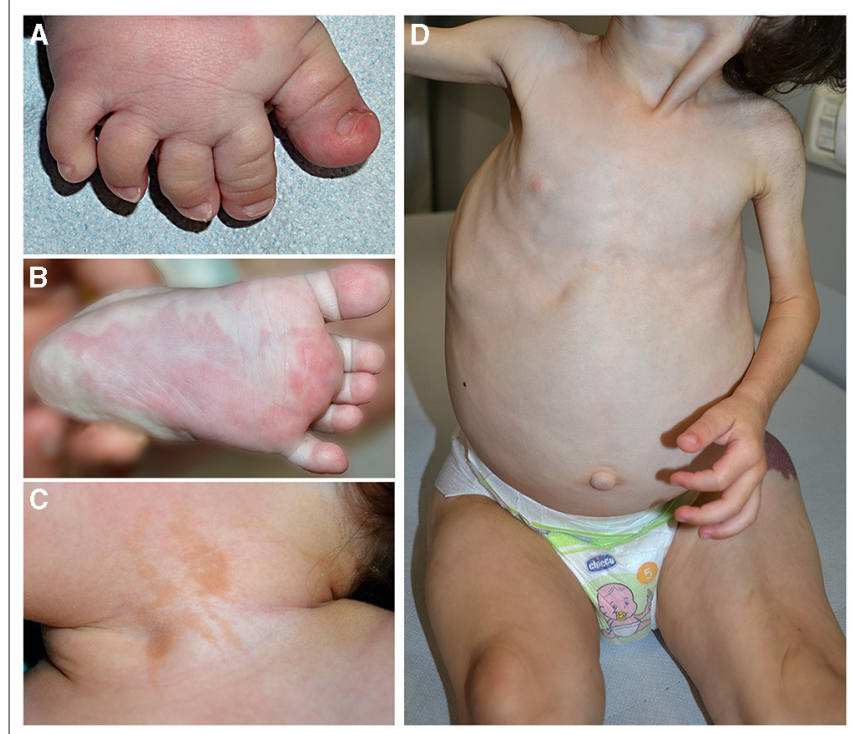

FIGURE 2 | Pseudosyndactyly (A), sandal gap, and capillary malformation in a patient with PIK3CA-related overgrowth syndrome (A). Triangular shape of the left foot (B) and epidermal nevus of the neck in the same patient (C). Plus/minus sign, overgrowth of lower limbs in contrast with upper limbs hypoplasia in a child affected with CLOVES (D).

localization depends on mosaic distribution but some areas are more frequently involved: the lateral aspect of the thigh in KTS (Figure 1C), the philtrum in MCAP (Figure 1D), the hip in CLOVES (Figure 1B), and the lower lip in CLAPO (Figure 1E). In MCAP and DCMO, CM is diffused with reticulated pattern and checkboard distribution (Figure 1F). CM due to PIK3CA mutations is commonly associated with lymphatic and/or venous malformations, which may manifest as grouped hyperkeratotic lymphangiectasias and prominent superficial veins (Figures 1B,C).

Clinical features frequently associated in PROS are pseudosyndactyly (Figure 2A), sandal gap, and triangular shape of the feet (Figures 2A,B). Epidermal nevus is a clinical sign of CLOVES syndrome, but it may be observed also in other PROS (11) (Figure 2C). In patients with MCAP, CM is associated with macrocrania. CLOVES and MCAP frequently manifest progressive overgrowth of lower limbs in contrast with upper limb hypoplasia (plus/minus sign) (Figure 2D).

Generalized overgrowth conditions associated with CM have increased risk to develop Wilms' tumor. However, the occurrence of malignancy in these patients is less frequent than in Beckwith-Wiedemann syndrome. Peterman et al. reported that Wilms tumor screening is recommended only in patients with hemihypertrophy and may be required in MCAP (15).

Capillary malformation arteriovenous malformation (CM$A V M)$. CM-AVM is a distinct entity; it is part of the arteriovenous malformations that derive from an altered vascular morphogenesis causing malformed arteries, veins, and capillaries with arteriovenous communications. CM-AVM has a prevalence of $1 / 100,000$ (16) characterized by several round-to-oval CM

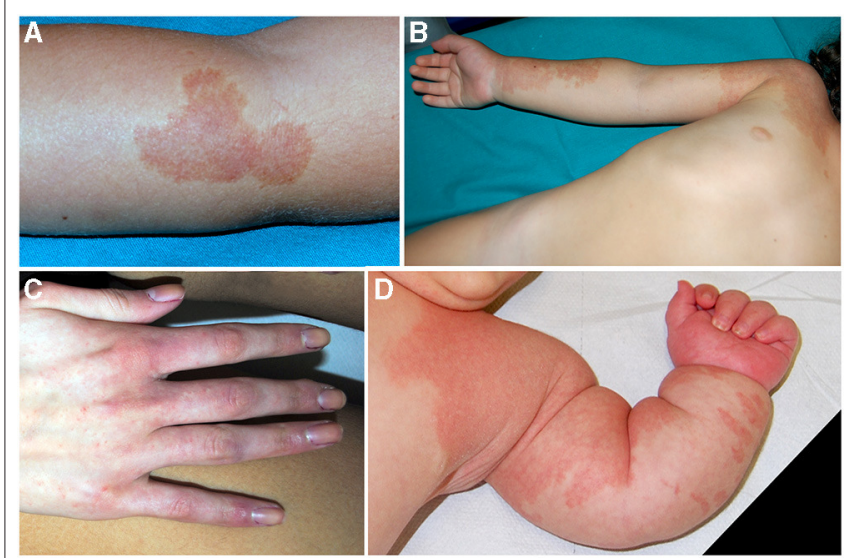

FIGURE 3 | Polycyclic vascular patch characterized by an anemic halo in a patient with CM-AVM (A). Pink-red slightly pigmented capillary malformation (CM) of the right upper limb (B). Pinpoint confluent telangiectasias in a patient with EPHB4 mutation (C). Eight-month-old infant affected with Parkes-Weber syndrome with multiple CM and progressive overgrowth of the upper limb (D).

with a diameter of few centimeters and a peculiar anemic halo (Figure 3A). The color of the lesions is pink, but they become hyperpigmented with time mimicking caffè-au-lait spots (Figure 3B). Indeed, on dermoscopy, CM lesions appear telangiectasic with a hyperpigmented background detectable after pressure (17). One-third of the affected patients presents arteriovenous fistulas (AVFs) in muscles, soft tissue, or CNS (18). These cases may carry pinpoint lesions that resemble common teleangiectasia, characterized by a typical whitish halo (Figure 3C), which is not present in Hereditary Hemorrhagic Teleangiectasia (HHT).

CM-AVM is an autosomal dominant syndrome due to mutations in RASA1 or EPHB4. Pathogenic variants in RASA1 and $E P H B 4$ have been identified in $\sim 50$ and $25 \%$ of the cases, respectively $(18,19)$. Germline loss-of-function mutations in EPHB4 causes a second form of CM-AVM (CM-AVM2) deregulating RAS-MAPK signaling (19).

Amyere and colleagues first described mutations in EPHB4 and proposed to distinguish between CM-AVM1 and CM-AVM2 caused by mutations in RASA1 and EPHB4. Telangiectasias distributed on hands, lips, and upper thorax are associated with mutation in EPHB4.

In patients in whom mutations have not been identified, the disease may be explained by genomic gene rearrangements, intronic mutations or, in regulatory regions, epigenetic factors or mosaicism (20). In the last condition, severity of the phenotype is not related to the level of mosaicism identified in blood (20). Moreover, the extreme intra-familiar variability of the syndrome is due to a two-hit mechanism as confirmed by the identification of germline and lesional somatic mutations in RASA1 (21).

Mutations in RASA1 may cause Parkes-Weber syndrome (PWS), which is part of the spectrum of RASA1-related diseases. In addition to multiple small CMs, patients affected with PWS present large CM associated with AVFs and progressive overgrowth of the involved limb (Figure 3D). 


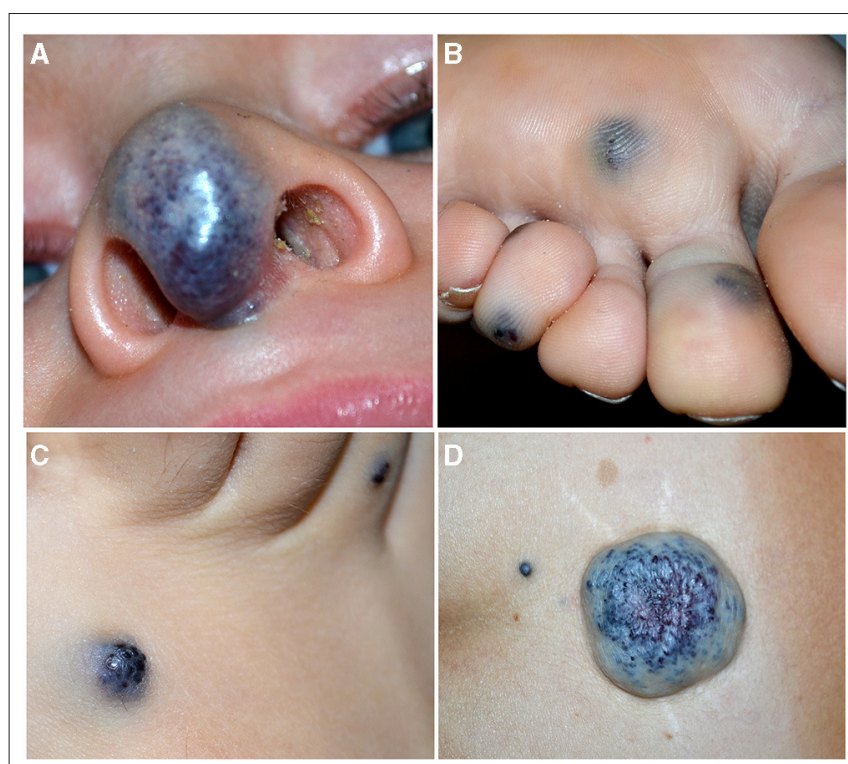

FIGURE 4 | Soft blue malformation of the tip of the nose in a toddler with a solitary venous malformation (VM) (A). Multiple small blue cutaneous VMs of the plantar (B) surface in a patient affected with blue rubber bleb nevus syndrome. Blue rubber bleb of the dorsal surface of the foot (C) and "dominant" congenital cutaneous VM of the lumbar area in the same patient (D).

The fast-flow vascular malformations may develop in the CNS, muscles, bones, and skin. Venous dilation in PWS patients is secondary to blood hyper-flow and not to venous valve insufficiency. For asymptomatic patients or for those with minor symptoms, conservative approach is recommended. Treatment decision should be evaluated by a multidisciplinary team. Complete excision is not usually possible, but in selected patients, catheter embolization may be combined with surgery (22). Multiple AVFs causing high-output cardiac failure require embolization and surgery.

\section{Venous Malformations}

Venous malformation (VM). It is the second common vascular malformation with an incidence of 1-5 in 10,000 births. They are slow-flow lesions, generally cutaneous unifocal and sporadic. They may be superficial or profound; when visible on the skin, they appear blue, soft, and compressible (Figure 4A). About 1$2 \%$ of VMs are multifocal and familial, and occur with a two-hit mechanism. They can develop in any part of the body, and the gastrointestinal tract is the most frequent visceral localization, which may manifest with hemorrhage and anemia.

Blue Rubber Bleb Nevus Syndrome (BRBNS). First described by Gascoyen in 1,860 , it is a rare sporadic condition caused by somatic double (cis) mutations in TEK gene (23). Patients present several VMs in the skin and/or visceral organs. BRBNS is characterized by gastrointestinal grape-like lesions visible on endoscopy, a "dominant" cutaneous or subcutaneous VM ( $>10$ times the other visible lesions), and numerous other small $(1-2 \mathrm{~cm})$ cutaneous VMs. Skin lesions are blue to purple in color, compressible, often hyperkeratotic, and mainly located in the palmoplantar areas, and they increase in size and number with time (24) (Figures 4B-D). Anemia due to recurrent gastrointestinal bleeding is the major complication of this syndrome.

\section{Lymphatic Malformations}

Micro and macrocystic lymphatic malformations (LM). They are congenital slow-flow malformations of lymphatic vessels with an incidence of 1.2-2.8 per 1,000 births. They are distinguished in micro-, macrocystic, or mixed depending on the dimension of the cysts, and occur within the first 2 years of life in $90 \%$ of the cases (25). They manifest as subcutaneous masses suddenly appearing or worsening after a minor infection, or with grouped transparent or blood-filled vesicles on the skin or oral mucosa. Disfigurement or compression of nearby structures with functional damage are the most important complications. In particular, $75 \%$ of LM is localized in the head and neck, with a predilection for submandibular region and parotid (Figure 5A) (26). Microcystic lymphangioma may involve the tongue and oral cavity causing macroglossia and related complications (sialorrhoea, dysphagia, and respiratory distress) (Figure 5B). The majority of the patients are initially asymptomatic; however, in more severe cases, tracheostomy and percutaneous endoscopic gastrostomy are needed to guarantee airway patency and adequate nutrition.

Excision of these malformations is usually not feasible due to the absence of clear margins, high risk of potential disfigurement, and functional impairment. Sclerotherapy is mostly indicated for macrocystic lesions, as isolated procedure or preceding surgery (27). In fact, surgical treatment has a high recurrence rate and combined therapy is required in most of the cases.

Gorham-Stout disease (GSD) and generalized lymphatic anomaly (GLA) are very rare LMs potentially involving all body sites, including the skeleton.

GSD is characterized by the presence of bone infiltration by microcystic lymphatic malformation with osteolysis and cortical bone resorption documented through computed tomography (CT). Patients manifest pathological fracture and pain and may have scoliosis and lymphorrhea and deformities (Figure 5C).

GLA is a multi-organ diffuse disease. Respiratory distress and bone fractures are the major complications in these patients. Pleural and pericardial effusion, rib and vertebral involvement, and young patient age are associated with a worse prognosis in GLA and GSD. Other localization may be the spleen, the peritoneum, and the gastrointestinal tract.

\section{Vascular Tumors}

IH is a benign vascular tumor characterized by three phases. It generally occurs after birth with a high proliferation during the first months of life, followed by a stabilization of the lesion at $\sim 9$ months of age and involution within 4-6 years of life (3).

They may be cutaneous, superficial, deep or mixed, and/or extracutaneous, focal, or multifocal. IH diameter ranges from a few millimeters to more than $5 \mathrm{~cm}$ (large lesions). Focal lesions have a round shape, while segmental IHs may involve one or more segments in a broad anatomic territory of skin (Figure 6A). Large segmental IHs may be associated 

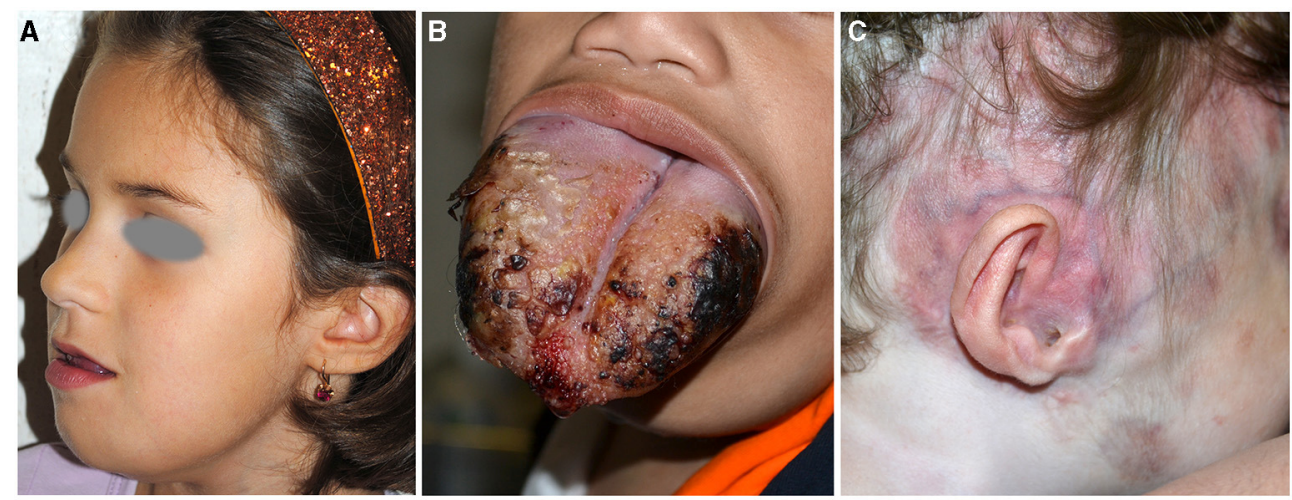

FIGURE 5 | Microcystic lymphangioma involving the tongue, the submandibular area, and the chin causing macroglossia, deformity, and sialorrhoea (A). Microcystic lymphangioma of the tongue in an 18-month-old patient treated with tracheostomy and percutaneous endoscopic gastrostomy for dysphagia and respiratory distress (B). CM and deformity of the auriculotemporal area due to osteolysis in a child affected with Gorham-Stout disease (C).
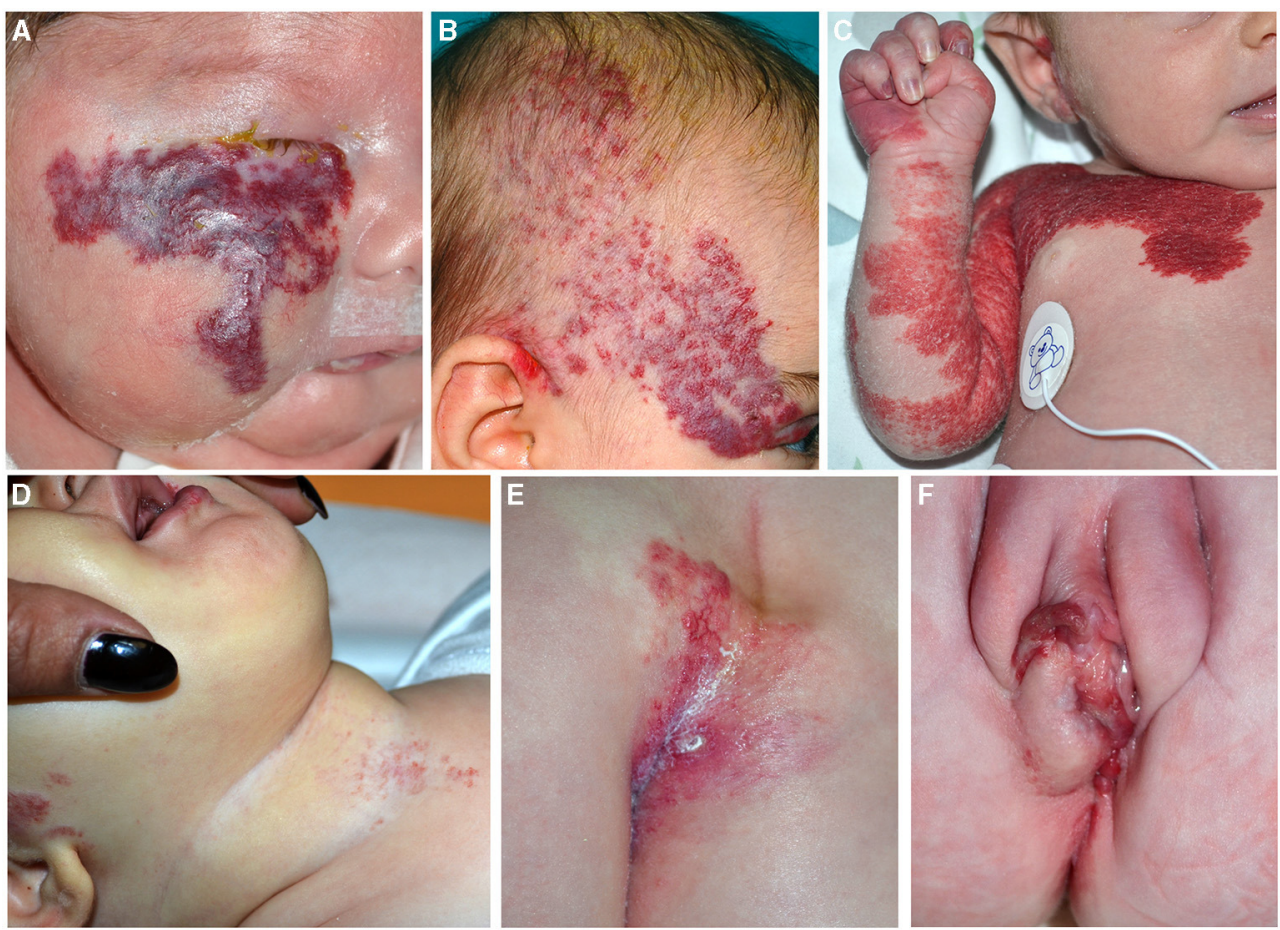

FIGURE 6 | Segmental infantile hemangiomas $(\mathrm{IH})$ of the face $\mathbf{( A , B ) ; ~ t h e ~ l e s i o n s ~ h a v e ~ a ~ d i a m e t e r ~ o f ~ t h a n ~} 5 \mathrm{~cm}$ and must be investigated for PHACE syndrome $\mathbf{( A ) .}$ Segmental $\mathrm{H}$ of the upper part of the trunk and the upper limb in a neonate (C). Segmental IH is on the beard area in an infant with obstruction of the upper airways (D). Segmental lumbar and sacral $\mathrm{IH}$ associated with myelopathy (E). $I \mathrm{H}$ of the vulva area associated with genital anomalies and imperforate anus (F).

with malformations of underlying structures in the same developmental field (3). Indeed, depending on the localization of the tumor, different syndromes have been described such as PHACE and LUMBAR/SACRAL/PELVIS.

PHACE syndrome includes posterior fossa malformations, hemangioma, arterial anomalies, coarctation of the aorta/cardiac defects, eye abnormalities, and sternal malformations. Not all these features are necessarily present to establish the diagnosis, which is based on specific criteria established in 2009 and modified in $2016(28,29)$. The IH should be large (more than $5 \mathrm{~cm}$ ) on the face (Figure 6B), but the syndrome may be present even with an IH of the upper part of the trunk and the upper limb or in the absence of IH if major criteria are present (Figure 6C). The syndrome has a prevalence of $2-3 \%$ of all IHs and 20$30 \%$ of large segmental IHs of the face (30). The female/male ratio is 9:1. Brain magnetic resonance imaging (MRI) findings lead to the stratification of low, intermediate, and high risk of a cerebrovascular accident in patients with PHACE syndrome (29). 
Based on the localization of the $\mathrm{IH}$, there is a different risk of extracutaneous involvement: the eye and brain in frontotemporal and frontonasal $\mathrm{IH}$, and the heart in mandibular lesions (31).

Other associated clinical features are headache, endocrine abnormalities, and auditory and dental anomalies. The beard pattern has been identified by the presence of $\mathrm{IH}$ in the right and left preauricular areas, lower lip, chin, and anterior neck. When segmental $\mathrm{IH}$ is distributed on the beard area, larynx can be involved, causing potentially life-threatening obstruction of the upper airways (Figure 6D). More rarely a subglottic IH may occur as a persistent croup-like illness in the absence of a cutaneous $\mathrm{IH}$ (32). In $50 \%$ of the patients affected with subglottic his, four or more areas were involved by the tumor, whereas $4 \%$ of cases had IH in one or two areas of the beard. The most frequently involved skin area in patients with subglottic $\mathrm{IH}$ is the mandibular (median) area (63\% of the cases in asymptomatic patients) $(33,34)$. Cutaneous IHs associated with subglottic involvement were significantly more telangiectatic than raised superficial lesions (33). Moreover, IHs of the beard area may be considered distributed in a segmental pattern and they are potentially associated with PHACE syndrome, as reported by Metry et al. (35). In addition, PHACE syndrome may be associated with subglottic IH even in the absence of beard IH (36).

Lower body $\mathrm{IH}$ and other skin defects, urogenital anomalies and ulceration, myelopathy, bony deformities, anorectal malformations, and arterial and renal anomalies (LUMBAR) syndrome occur when a large segmental IH is localized in the lower part of the body in association with extracutaneous regional malformations (Figures 6E,F). This acronym is now considered more comprehensive of the malformations that can be associated with $\mathrm{IH}$ in the lower part of the body, replacing the terms spinal dysraphism, anogenital, cutaneous, renal and urologic anomalies, associated with an $\mathrm{IH}$ of lumbosacral localization (SACRAL) and perineal hemangioma, malformations of external genitalia, lipomyelomeningocele, vesicorenal abnormalities, imperforate anus and skin tag (PELVIS) syndrome (37).

The most common localization of $\mathrm{IH}$ is the sacral region followed by the lumbar, perineum, and the lower limbs. Segmental IH located in the sacral region may be associated with urogenital and anorectal malformations, while IH in the lumbar area may be associated with myelopathy. Ulceration is frequent when more than three regions are affected (37).

Tufted angioma (TA). It is a benign vascular tumor whose name derives from the "tufts of hypertrophied endothelial cells" (38) in the dermis with a cannonball distribution. TA may be congenital or acquired in the first years of life. It is usually a solitary red purple plaque or nodule, but multifocal cases have been reported (Figure 7A). Most of the cases are asymptomatic; pain may be present. In a minority of the cases, KasabachMerritt phenomenon (KMP) may be associated. It is frequently distributed in the head and neck or in the upper trunk, but other localizations are described. Even if spontaneous regression may occur, persistent lesions are frequently painful and treatment is needed (39).

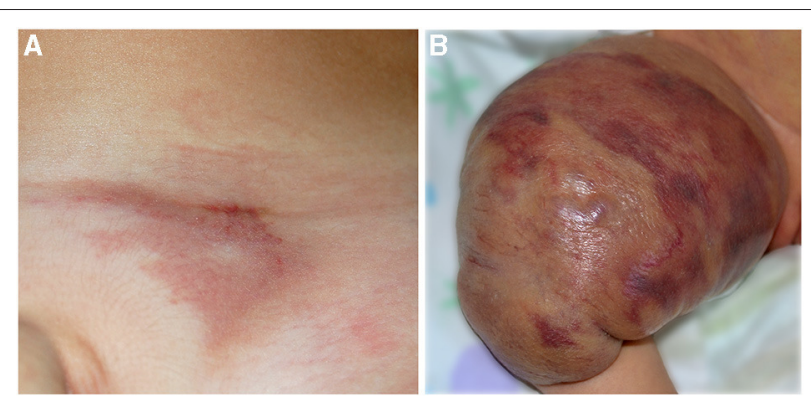

FIGURE 7 | Red vascular plaque of the pubic area in a toddler (A); the lesion is painful and warm. Voluminous vascular tumor characterized by red brilliant purpuric color associated with the Kasabach-Merritt phenomenon in a newborn (B).

Kaposiform hemangioendothelioma (KHE). KHE is a rare locally aggressive vascular tumor characterized by the presence of spindle cells with a positive staining for D2-40 lymphatic marker. On histology, KHE has an infiltrative behavior and may affect tissues, muscles, and bones with possible visceral involvement (mediastinum and retroperitoneum). The onset is at birth or in infancy presenting as a reddish plaque or multiple nodules (Figure 7B). In the majority of cases (42-71\%), $\mathrm{KHE}$ is associated with the KMP (40). It is a life-threatening consumptive coagulopathy causing severe thrombocytopenia, together with hypofibrinogenemia (41). When KMP occurs, the lesion becomes swollen, hot, and purpuric with a relevant enlargement. Combined treatments are warranted for KMP, and platelet transfusion should be performed only in case of bleeding or prior to surgery (40). Apart from KMP, other complications are possible in KHE: musculoskeletal disorders, lymphedema, and compression of vital structures (40). The diagnosis is made on histology, but MRI is necessary to define margins and deepness of the tumor (42).

\section{DISCUSSION}

Consultations for vascular birthmarks are very common in dermatology, given the high rate of vascular lesions in the neonatal period. The vast majority of the cutaneous manifestations are transient or benign (salmon patch, nevus simplex, and stork bite) and reassuring the parents is sufficient. The assessment of the patients should start from a complete clinical history (family, pregnancy, and personal history of the mother and the child). Indeed, some vascular malformations such as multifocal VM and CM-AVM may recur in the family, while $\mathrm{IH}$ is associated to low birthweight, in vitro fertilization, prematurity, and twin pregnancy $(3,43,44)$.

It is very important to determine if the vascular anomaly was present at birth or developed in the first days/weeks of life. Vascular malformations are present at birth, but sometimes deep venous and lymphatic malformations may become visible after birth because of thrombosis or swelling, respectively. On the other hand, IHs occur in the first weeks of life sometimes preceded by an anemic patch, while congenital hemangiomas are 

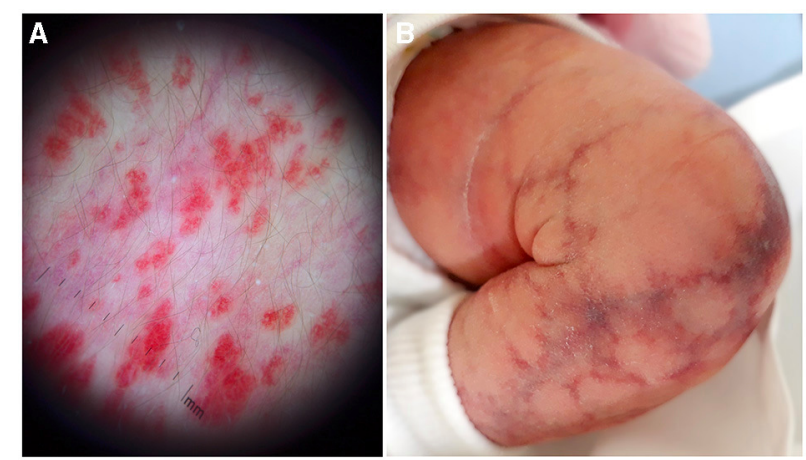

FIGURE 8 | Dermoscopy of a flat infantile hemangioma shows a micro-papular pattern and allows to differentiate it from capillary malformation (A). Cutis marmorata telangiectatica congenital with the typical reticulated linear atrophy (B).

always present at birth and do not increase in size. In addition, congenital hemangiomas do not have risk factors like IHs. If precise documentation of the lesion at birth is not available, it is useful asking the parents to show photographic images of the newborn.

In the presence of a superficial vascular anomaly, the differential diagnosis between vascular malformations and vascular tumor is easy for an expert dermatologist, according to the ISSVA classification updated in 2018. Rarely, IHs may present as a flat vascular patch; dermoscopy highlights the micropapular pattern of the lesion and allows to differentiate it from CM (Figures 6B, 8A). CMs appear as a flat homogeneous red patch with sharp margins. VMs manifest as soft blue masses on the skin or mucous membranes that typically enlarge when in declive position. Microcystic lymphatic malformation may be superficial, emerging on the skin as grouped vesicles with transparent or hematic content. These lesions may ooze or bleed especially in summer.

The extension of the vascular malformation may correlate with severity of the disease. Indeed, the wider is the diffusion of the malformation, the earlier must be the mutation responsible for the phenotype, with a wider involvement of tissue and organs. In general, localization of vascular birthmark on the head may be associated with cerebral involvement. The main variables that determine the phenotype in mosaic disorders is the mutation, the normal function of the gene, the timing of the mutation during embryogenesis, and the destiny of the mutated cell. Indeed, different clinical results may be produced by an identical genetic defect (45).

Clinical examination should always be complete and not limited to the vascular anomaly. Indeed, in particular with $\mathrm{CM}$, many other cutaneous or extracutaneous signs should be investigated. The presence of other nevoid lesions may orientate the diagnosis: an epidermal nevus associated with CMs may suggest a PROS, while a large nevus spilus may suggest a phacomatosis spilorosea.

Macrocephaly together with CMs may be a clue for the diagnosis of megalencephaly-CM or PTEN hamartoma tumor syndrome. Pseudosyndactyly, sandal gap sign, and limb overgrowth may be associated with CM and overgrowth in PROS. The presence of a vascular anomaly, segmental $\mathrm{IH}$ or a CM, in the lumbar and sacral region suggests the presence of associated malformation: bone deformities, anorectal malformations, lipomatous masses, spinal dysraphism, urogenital, and renal malformations.

Doppler-ultrasound is the examination of first choice for suspected deep vascular anomaly (46). Indeed, this non-invasive technique usually allows the radiologist to identify a vascular lesion, to distinguish between tumor and malformation, and to confirm a slow-flow or fast-flow vascular anomaly. Nevertheless, newborns and infants do not collaborate and cry, producing a lot of artifacts. For this reason, based on our clinical experience, we tell the mother that the baby should be fasted and to breastfeed or to give the feeding bottle in the waiting room to help the newborn to sleep or during the exam in order to improve the quality of the examination.

Differential diagnosis of deep vascular lesions is between vascular tumors and malformations but also with non-vascular malignant tumor. For this reason, when the clinical history, together with the clinical characteristics, and the Dopplerultrasound are not diagnostic, a biopsy is mandatory. In some cases, the surgeon requires magnetic resonance imaging before the procedure in order to investigate the margins of the lesion and its relationship with surrounding structures (47).

ISSVA classification represents an important step forward that has provided a common language for the scientific community dealing with vascular anomalies. Nevertheless, with the aim of schematizing and relate different clinical pictures to a single recognized entity, there is a risk of oversimplifying. It is the case of CMs that have been defined as slow-flow CMs but are included in the definition of CM-AVM, a disease that is known to be a fast-flow vascular malformation. CMs in CM-AVM are usually multiple, and they have a peculiar clinical feature: small red pink color patches with round margins and a white anemic halo, and they are warm. Sometimes, it is possible to appreciate a bruit or a trill underneath. The difference between slow-flow CMs and CMs in CM-AVM has been demonstrated by Valdivielso-Ramos $\mathrm{M}$ et al., who suggest that they histologically and immunohistochemically resemble an incipient arteriovenous malformation (48). In addition, CMs in CM-AVM are familial, while simple CMs are not.

Moreover, CM due to GNAQ mutation has a different appearance from CM observed in PROS. Indeed, the latter exhibits a more geographical margin, a dark red color, and may present lymphangiectasias on the surface and an underlying prominent venous reticulum. Normally, hypertrophy of soft tissue due to GNAQ mutation, if present, is lower than the one due to PIK3CA mutation, which is typically progressive. When CM is located in the frontonasal area, the eye and the pia mater may be involved due to an early mutation in the prosencephalon. The patient may be affected with SWS and presents epilepsy and/or glaucoma. Controversies are reported in the literature on when to carry out brain MRI in asymptomatic patients who present CM potentially associated with SWS. An exhaustive examination is possible only after the age of 1 year due 
to maturation of brain. In the absence of neurological symptoms, it is recommended to postpone brain MRI in general anesthesia with gadolinium to the second year of life (49). Brain MRI during sleep is possible in the first months of life but normal results do not rule out SWS.

CMs in phacomatosis spilorosea have a characteristic pink color and have been recently associated with mutation in PTPN11 (6). Cutis marmorata telangiectatica congenita is actually classified as a CM, but has a reticulated pattern with a peculiar linear atrophy and no mutations have been yet identified in this malformation (Figure 8B). In addition, the evolution is completely different: ulceration is possible, but the lesions improve enormously in the first 6 months of life, while CMs are usually stable

VMs are distinguished into common VM, familial CutaneoMucosal VM (VMCM), glomuvenous malformation (GVM), Blue Rubber Bleb Nevus syndrome (BRBNS), and cerebral cavernous malformation (CCM). In case of a venous malformation, accurate family history to rule out a familial condition is mandatory as well as a full blood count and fecal occult blood test. Indeed, single cutaneous lesions have also been recently reported in patients with intestinal involvement, which may manifest with bleeding, requiring an emergency treatment (50).

Moreover, VM is generally present at birth, but multiple lesions may develop later in life due to a two-hit mechanism in the TEK gene (23).

GMVs are easily diagnosed; they manifest with multiple cutaneous blue macules or papules, sometimes grouped in patches and painful on pressure. They are due to a mutation in the glomulin (GLMN) gene.

BRBNS may be suspected in the presence of a "dominant" congenital cutaneous lesion associated with multiple compressible, often hyperkeratotic, small skin lesions frequently located on palmoplantar surfaces. Additional VMs appear during life and gastrointestinal lesions may cause severe anemia.

Lymphatic malformations are localized in the head and neck in $75 \%$ of cases and frequently involve deep and vital structures. They should be suspected in patients who manifest sudden swelling of the neck or parotid in the first years of life. A careful observation of the oral mucosa may identify typical grouped vesicle of the microcystic lymphangioma. In more severe cases, macroglossia and obstructive respiratory symptoms are present. Diagnosis may be easily confirmed with ultrasound but magnetic resonance is mandatory to study the extension of the malformation and its relation with the neighbor structures. In case of obstruction of the upper airways, laryngoscopy is mandatory in order to evaluate the percentage of obstruction and to discuss the necessity of a tracheostomy.

Concerning vascular tumors, it is known that 10$12 \%$ of $\mathrm{IHs}$ may require treatment due to ulceration, life threatening complications, functional damage, and permanent disfigurement.

In case of a segmental and large $\mathrm{IH}$, it is mandatory to rule out associated malformations when the tumor is located in the head, neck, arm and upper trunk or in the lower part of the body. When PHACE syndrome is suspected, brain MRI, ophthalmologic evaluation, and echocardiography should be performed. In case of lower body large and segmental $\mathrm{IH}$, or IH on the median line, LUMBAR syndrome should be ruled out with spinal and pelvic MRI and abdominal ultrasound (51). In addition, in newborns with large IH, or with multiple liver IHs, it is necessary to investigate hypothyroidism.

Congenital hemangiomas are usually easily distinguished due to their clinical characteristics. They are frequently located on head and neck and extremities. RICH has a dome shape with a pale rim and a central depression while $\mathrm{NICH}$ is slightly elevated with a blue hue covered by thick telangiectasias and with a peripheral pallor. They are large at birth and never increase in size; NICH stably persists, while RICH is rapidly involved within the first 18 months of life or partially regresses (PICH). Congenital hemangiomas are not a sign of a complex disorder; however, they should be considered in differential diagnosis with other vascular tumors and neoplastic disorders.

When an angiomatous mass of a newborn is associated with thrombocytopenia, KMP should be suspected. KMP occurs in TA and KHE that may be congenital or appear after birth. Rarely, $\mathrm{RICH}$ may be associated with a transient thrombocytopenia. Patients with TA or KHE should be regularly investigated in the first 6 months of life. In KMP, platelets are very low, fibrinogen is low, and $\mathrm{D}$-dimers are very high, but it has been a common experience of the experts that these patients do not manifest spontaneous hemorrhages as expected based on the laboratory values. Moreover, in KMP, platelets should never be transfused with the exception of bleeding or immediately prior to surgery. Steroids and acetylsalicylic acid should be immediately started, and biopsy is suggested in order to confirm the diagnosis and to start II level treatment with sirolimus or vincristine. KMP is present only in the first months, and then, after treatment and regression, the vascular tumor may present as a pseudo-CM, with telangiectatic streaks and swelling or with a sclerodermalike plaque.

TA usually presents as a reddish-brownish single or multiple patch sometimes infiltrated with hyperidrosis or hypertrichosis. KHE is frequently deeper and may infiltrate the mediastinum and retroperitoneum sometimes involving the skin as a reddishviolaceous plaque or a voluminous mass.

In the recent years, many advancements have been achieved in the understanding of the molecular mechanism and genetic causes of vascular anomalies. While mutated genes in familial vascular anomalies are vascular cell-type specific, genes mutated in sporadic vascular malformations are widely expressed in all tissues. The RAS/MAP/ERK and the PI3K/AKT/mTOR signaling pathways are regulators of cell proliferation, migrations, and apoptosis, and are involved in oncogenesis. Mutation in these two pathways is responsible for the vast majority of vascular malformations (52).

It is now clear that multiple entities are part of wide spectrum of possibilities depending on the mutated gene, site, and timing of the mutation. For example, PROS includes different phenotypes with variable severity, from macrodactyly to CLOVES syndrome. Moreover, identification of causative genes of the vast majority of vascular malformations has opened up the possibility of repurposing anticancer molecules as new target therapies for 
vascular malformations. Sirolimus, a mTOR inhibitor, has shown promising results in the management of complicated vascular anomalies $(53,54)$. In addition, new molecules such as Alpelisib, a PIK3CA inhibitor, and Miransertib, an AKT inhibitor, are nowadays the subject of clinical trials (55). Moreover, MEK inhibitors and Vemurafenib have restored vascular anomalies in a zebrafish model of EPHB4, BRAF, and MAP2K1 mutations, respectively (56).

Biopsy of superficial lesions is a very simple and fast procedure, but it requires general anesthesia in children below the age of 6 years. On the contrary, in the first 2 years of life, the child is not fully aware of what happens around him and it can be easy to collect a few millimeters of a sample of affected tissue. For this reason, we suggest, in more severe cases who may be candidates for a target therapy, for the child to immediately undergo genetic testing. Recently, cell-free DNA next-generation sequencing liquid biopsy, a technique derived from oncologic settings, has demonstrated to be an effective tool for KTS diagnosis, opening a new possibility for other mosaic vascular anomalies as a non-invasive diagnostic approach (57). In addition, in patients with phenotype involving the head or the brain, NGS from buccal swab demonstrated a sensitivity comparable to NGS from tissue biopsies. In these patients, the detection rate of the pathogenic or likely pathogenic mutation from buccal swab increases to $57 \%$ (58). Altogether, these new techniques will allow to perform more diffusely genetic testing in newborns and infants, in order to early detect the pathogenic mutations responsible for the phenotype.

In conclusion, cutaneous birthmarks may be an important clue for the diagnosis of complex and syndromic vascular anomalies but is often underestimated. They represent a phenotypic and genotypic heterogeneous wide group of

\section{REFERENCES}

1. Dasgupta R, Fishman SJ. ISSVA classification. Semin Pediatr Surg. (2014) 23:158-61. doi: 10.1053/j.sempedsurg.2014.06.016

2. Techasatian L, Sanaphay V, Paopongsawan P, Schachner LA. Neonatal birthmarks: a prospective survey in 1000 neonates. Glob Pediatr Health. (2019). 6, 1-8. doi: 10.1177/2333794X19835668

3. Léauté-Labrèze C, Harper JI, Hoeger PH. Infantile haemangioma. Lancet. (2017) 390:85-94. doi: 10.1016/S0140-6736(16)00645-0

4. Waelchli R, Aylett SE, Robinson K, Chong WK, Martinez AE, Kinsler VA. New vascular classification of port-wine stains: improving prediction of Sturge-Weber risk. Br J Dermatol. (2014) 171:861-7. doi: 10.1111/bjd.13203

5. Dutkiewicz A, Ezzedine K, Mazereeuw-Hautier J, Lacour J, Barbarot S, Vabres $\mathrm{P}$, et al. A prospective study of risk for Sturge-Weber syndrome in children with upper facial port-wine stain. J Am Acad Dermatol. (2015) 72:473-80. doi: 10.1016/j.jaad.2014.11.009

6. Polubothu S, Al-Olabi L, del Boente MC, Chacko A, Eleftheriou G, Glover $\mathrm{M}$, et al. GNA11 mutation as a cause of sturge-weber syndrome: expansion of the phenotypic spectrum of $\mathrm{G} \alpha / 11$ mosaicism and the associated clinical diagnoses. J Invest Dermatol. (2020) 140:1110-3. doi: 10.1016/j.jid.2019.10.019

7. Thorpe J, Frelin LP, McCann M, Pardo CA, Cohen BA, Comi AM, et al. Identification of a mosaic activating mutation in GNA11 in atypical sturge-weber syndrome. J Invest Dermatol. (2021) 141:685-8. doi: $10.1016 /$ j.jid.2020.03.978 frequently disabling disorders with potentially severe and life-threatening complications. Even if recent advancement in genetics allowed better understanding of the pathogenesis of these diseases and their classification, controversies are still present and management is not yet fully standardized. Finally, in selected cases, genetic testing should be rapidly performed in order to confirm the diagnosis and to offer genetic counseling; identification of the mutated gene may offer the opportunity to undergo target therapy and to be enrolled in clinical trials. For these reasons, patients should be promptly referred to a multidisciplinary reference center for appropriate management and follow-up.

\section{AUTHOR CONTRIBUTIONS}

$\mathrm{AD}$ and $\mathrm{ME}$ : conceptualization, project administration, and supervision. AD, GP, MZ, RA, CCa, AC, CCe, MD, SB, and MR: investigation. GP, MZ, RA, and MR: visualization. $\mathrm{AD}$, $\mathrm{CCa}$, and $\mathrm{ME}$ : writing-original draft. $\mathrm{AD}, \mathrm{GP}, \mathrm{MZ}, \mathrm{RA}$, $\mathrm{CCa}, \mathrm{AC}, \mathrm{CCe}, \mathrm{MD}, \mathrm{SB}, \mathrm{MR}$, and $\mathrm{ME}$ : writing-review and editing. All authors contributed to the article and approved the submitted version.

\section{ACKNOWLEDGMENTS}

We thank patients' parents for accepting to share pictures and data of their children, and Gabriele Bacile for iconography preparation. $\mathrm{AD}$ and $\mathrm{ME}$ are members of the European Reference Network for Rare Skin Disease/ERN-Skin and Vascular Anomalies Working Group of the European Reference Network for Rare Multisystemic Vascular Diseases (VASCERN).

8. Shirley MD, Tang H, Gallione CJ, Baugher JD, Frelin LP, Cohen B, et al. Sturge-weber syndrome and port-wine stains caused by somatic mutation in GNAQ. N Engl J Med. (2013) 368:1971-9. doi: 10.1056/NEJMoa1213507

9. Queisser A, Boon LM, Vikkula M. Etiology and genetics of congenital vascular lesions. Otolaryngol Clin North Am. (2018) 51:41-53. doi: 10.1016/j.otc.2017.09.006

10. Ayturk UM, Couto JA, Hann S, Mulliken JB, Williams KL, Yue Huang A, et al. Somatic activating mutations in GNAQ and GNA11 are associated with congenital hemangioma. Am J Hum Genet. (2016) 98:789-95. doi: 10.1016/j.ajhg.2016.05.010

11. Keppler-Noreuil KM, Rios JJ, Parker VE, Semple RK, Lindhurst MJ, Sapp JC, et al. PIK3CA-related overgrowth spectrum (PROS): diagnostic and testing eligibility criteria, differential diagnosis, and evaluation. Am J Med Genet A. (2015) 167A:287-95. doi: 10.1002/ajmg.a.36836

12. Vahidnezhad H, Youssefian L, Uitto J. Klippel-Trenaunay syndrome belongs to the PIK3CA-related overgrowth spectrum (PROS). Exp Dermatol. (2016) 25:17-9. doi: 10.1111/exd.12826

13. Downey C, López-Gutiérrez JC, Roé-Crespo E, Puig L, Baselga E. Lower lip capillary malformation associated with lymphatic malformation without overgrowth: part of the spectrum of CLAPO syndrome. Pediatr Dermatol. (2018) 35:e243-4. doi: 10.1111/pde.13514

14. Goss JA, Konczyk DJ, Smits P, Sudduth CL, Bischoff J, Liang MG, et al. Diffuse capillary malformation with overgrowth contains somatic PIK3CA variants. Clin Genet. (2020) 97:736-40. doi: 10.1111/cge.13702 
15. Peterman CM, Fevurly RD, Alomari AI, Trenor CC 3rd, Adams DM, Vadeboncoeur S, et al. Sonographic screening for Wilms tumor in children with CLOVES syndrome. Pediatr Blood Cancer. (2017) 64. 1-7. doi: $10.1002 /$ pbc. 26684

16. Revencu N, Boon LM, Mulliken JB, Enjolras O, Cordisco MR, Burrowset $\mathrm{PE}$, et al. Parkes Weber syndrome, vein of Galen aneurysmal malformation, and other fast-flow vascular anomalies are caused by RASA1 mutations. Hum Mutat. (2008) 29:959-65. doi: 10.1002/humu.20746

17. Edwards LR, Blechman AB, Zlotoff BJ. RASA1 mutation in a family with capillary malformation-arteriovenous malformation syndrome: a discussion of the differential diagnosis. Pediatr Dermatol. (2018) 35:e9-12. doi: $10.1111 /$ pde. 13332

18. Revencu N, Boon LM, Mendola A, Cordisco MR, Dubois J, Clapuyt P, et al. RASA1 mutations and associated phenotypes in 68 families with capillary malformation- arteriovenous malformation. Hum Mutat. (2013) 34:1632-41. doi: 10.1002/humu.22431

19. Amyere M, Revencu N, Helaers R, Pairet E, Baselga E, Cordisco M. Germline loss-of-function mutations in EPHB4 cause a second form of capillary malformation arteriovenous malformation (CM-AVM2) deregulating RAS-MAPK signaling. Circulation. (2017) 12; 136:1037-48. doi: 10.1161/CIRCULATIONAHA.116.026886

20. Revencu N, Fastre E, Ravoet M, Helaers R, Brouillard P, BisdorffBresson A, et al. RASA1 mosaic mutations in patients with capillary malformation-arteriovenous malformation. J Med Genet. (2020) 57:48-52. doi: 10.1136/jmedgenet-2019-106024

21. Macmurdo CF, Wooderchak-Donahue W, Bayrak-Toydemir P, Le J, Wallenstein MB, Millaet $\mathrm{C}$, et al. RASA1 somatic mutation and variable expressivity in capillary malfor- mation/arteriovenous malformation (CM/AVM) syndrome. Am J Med Genet A. (2016) 170:1450-4. doi: 10.1002/ajmg.a.37613

22. Conway AM, Qato K, Nguyen Tran NT, Short H, Giangola G, Carroccio A. Embolization techniques for arteriovenous malformations in parkes-weber syndrome. Ann Vasc Surg. (2020) 69:224-31. doi: 10.1016/j.avsg.2020.05.039

23. Soblet J, Kangas J, Nätynki M, Mendola A, Helaers R, Uebelhoer M. Blue rubber bleb nevus (BRBN) syndrome is caused by somatic TEK (TIE2) mutations. J Invest Dermatol. (2017) 137:207-16. doi: 10.1016/j.jid.2016.07.034

24. Ballieux F, Boon LM, Vikkula M. Blue bleb rubber nevus syndrome. Handb Clin Neurol. (2015) 132:223-30. doi: 10.1016/B978-0-444-62702-5.00016-0

25. Lerat J, Mounayer C, Scomparin A, Orsel S, Bessede JP, Aubry K. Head and neck lymphatic malformation and treatment: clinical study of 23 cases. Eur Ann Otorhinolaryngol Head Neck Dis. (2016) 133:393-6. doi: 10.1016/j.anorl.2016.07.004

26. Grasso DL, Pelizzo G, Zocconi E, Schleef J. Lymphangiomas of the head and neck in children. Acta Otorhinolaryngol Ital. (2008) 28:17-20.

27. De Maria L, De Sanctis P, Balakrishnan K, Tollefson M, Brinjikji W. Sclerotherapy for lymphatic malformations of head and neck: systematic review and meta-analysis. J Vasc Surg Venous Lymphat Disord. (2020) 8:15464. doi: 10.1016/j.jvsv.2019.09.007

28. Metry D, Heyer G, Hess C, Garzon M, Haggstrom A, Frommelt P. PHACE syndrome research conference. Consensus statement on diagnostic criteria for PHACE syndrome. Pediatrics. (2009) 124:1447-56. doi: 10.1542/peds.2009-0082

29. Garzon MC, Epstein LG, Heyer GL, Frommelt PC, Orbach DB, Baylis AL, et al. PHACE syndrome: consensus-derived diagnosis and care recommendations. J Pediatr. (2016) 78:24-33.e2. doi: 10.1016/j.jpeds.2016.07.054

30. Rotter A, Samorano LP, Rivitti-Machado MC, Oliveira ZNP, Gontijo B. PHACE syndrome: clinical manifestations, diagnostic criteria, and management. An Bras Dermatol. (2018) 93:405-11. doi: 10.1590/abd1806-4841.20187693

31. Haggstrom AN, Lammer EJ, Schneider RA, Marcucio R, Frieden IJ. Patterns of infantile hemangiomas: new clues to hemangioma pathogenesis and embryonic facial development. Pediatrics. (2006) 117:698-703 doi: 10.1542/peds.2005-1092

32. O-Lee TJ, Messner A. Subglottic hemangioma. Otalaryngol Clin Noarth Am. (2008) 41:903-11, viii-ix. doi: 10.1016/j.otc.2008.04.009

33. Piram M, Hadj-Rabia S, Boccara O, Couloigner V, Hamel-Teillac D, Bodemer C. Beard infantile hemangioma and subglottic involvement: are median pattern and telangiectatic aspect the clue? J Eur Acad Dermatol Venereol. (2016) 30:2056-9. doi: 10.1111/jdv.13812

34. Orlow SJ, Isakoff MS, Blei F. Increased risk of symptomatic hemangiomas of the airway in association with cutaneous hemangiomas in a "beard" distribution. J Pediatr. (1997) 131:643-6. doi: 10.1016/S0022-3476(97)70079-9

35. Metry DW, Dowd CF, Barkovich AJ, Frieden IJ. The many faces of PHACE syndrome. J Pediatr. (2001) 139:117-23. doi: 10.1067/mpd.2001.114880

36. Smith DS, Lee KK, Milczuk HA. Otolaryngologic manifestations of PHACE syndrome. Int J Pediatr Otorhinolaryngol. (2004) 68:1445-50. doi: 10.1016/j.ijporl.2004.05.013

37. Yu X, Zhang J, Wu Z, Liu M, Chen R, Gu Y. LUMBAR syndrome: a case manifesting as cutaneous infantile hemangiomas of the lower extremity, perineum and gluteal region, and a review of published work. J Dermatol. (2017) 44:808-12. doi: 10.1111/1346-8138.13763

38. Jones EW, Orkin M. Tufted angioma (angioblastoma). A benign progressive angioma, not to be confused with Kaposi's sarcoma or low-grade angiosarcoma. J Am Acad Dermatol. (1989) 20:214-25. doi: 10.1016/S0190-9622(89)70025-6

39. Häußler SM, Uecker FC, Knopke S, Roßner F, Olze H, Böttcher A. Tufted angiomas of the head and neck. HNO. (2017) 65:981-6. doi: 10.1007/s00106-017-0379-z

40. Ji Y, Chen S, Yang K, Xia C, Li L. Kaposiform hemangioendothelioma: current knowledge and future perspectives. Orphanet J Rare Dis. (2020) 15:39. doi: 10.1186/s13023-020-1320-1

41. Adams DM, Brandão LR, Peterman CM, Gupta A, Patel M, Fishman S, et al. Vascular anomaly cases for the pediatric hematologist oncologistsAn interdisciplinary review. Pediatr Blood Cancer. (2018) 65. 1-9. doi: $10.1002 /$ pbc.26716

42. Peng S, Xia C, Yang K, Chen S, Ji Y. Kaposiform haemangioendothelioma: magnetic resonance imaging features in 64 cases. BMC Pediatr. (2021) 21:107. doi: 10.1186/s12887-021-02573-8

43. Hoeger PH. Infantile haemangioma: new aspects on the pathogenesis of the most common skin tumour in children. Br J Dermatol. (2011) 164:234-5. doi: 10.1111/j.1365-2133.2011.10204.x

44. Hoeger PH, Harper JI, Baselga E, Bonnet D, Boon LM, Ciofi Degli Atti M, et al. Treatment of infantile haemangiomas: recommendations of a European expert group. Eur J Pediatr. (2015) 174:855-65. doi: 10.1007/s00431-015-2570-0

45. Kinsler VA, Boccara O, Fraitag S, Torrelo A, Vabres P, Diociaiuti A. Mosaic abnormalities of the skin: review and guidelines from the European reference network for rare skin diseases. Br J Dermatol. (2020) 182:552-63. doi: 10.1111/bjd.17924

46. Ding A, Gong X, Li J, Xiong P. Role of ultrasound in diagnosis and differential diagnosis of deep infantile hemangioma and venous malformation. $J$ Vasc Surg Venous Lymphat Disord. (2019) 7:715-23. doi: 10.1016/j.jvsv.2019. 01.065

47. Bertino F, Trofimova AV, Gilyard SN, Hawkins CM. Vascular anomalies of the head and neck: diagnosis and treatment. Pediatr Radiol. (2021). 51. 1162-84. doi: 10.1007/s00247-021-04968-2

48. Valdivielso-Ramos M, Torrelo A, Martin-Santiago A, Hernández-Nuñez A, Azaña JM, Campos M, et al. Histopathological hallmarks of cutaneous lesions of capillary malformation-arteriovenous malformation syndrome. J Eur Acad Dermatol Venereol. (2020) 34:2428-35. doi: 10.1111/jdv.16326

49. De la Torre AJ, Luat AF, Juhász C, Ho ML, Argersinger DP, Cavuoto $\mathrm{KM}$, et al. A multidisciplinary consensus for clinical care and research needs for sturge-weber syndrome. Pediatr Neurol. (2018) 84:11-20. doi: 10.1016/j.pediatrneurol.2018.04.005

50. Diociaiuti A, Rotunno R, Caldaro T, Rossi S, Carnevale C, Torroni F, et al. Cutaneous venous malformations as a clue for possible gastrointestinal tract involvement: diagnosis and treatment of six cases. Dermatol Ther. (2021) e14932. 34. doi: 10.1111/dth.14932

51. Schumacher WE, Drolet BA, Maheshwari M, Horii KA, Nopper AJ, Newell $\mathrm{BD}$, et al. Spinal dysraphism associated with the cutaneous lumbosacral infantile hemangioma: a neuroradiological review. Pediatr Radiol. (2012) 42:315-20. doi: 10.1007/s00247-011-2262-5

52. Dekeuleneer V, Seront E, Van Damme A, Boon LM, Vikkula M. Theranostic advances in vascular malformations. J Invest Dermatol. (2020) 140:756-63. doi: 10.1016/j.jid.2019.10.001 
53. Isoldi S, Belsha D, Yeop I, Uc A, Zevit $\mathrm{N}$, Mamula $\mathrm{P}$, et al. Diagnosis and management of children with blue rubber bleb nevus syndrome: a multi-center case series. Dig Liver Dis. (2019) 51:1537-46. doi: 10.1016/j.dld.2019.04.020

54. Wong XL, Phan K, Rodríguez Bandera AI, Sebaratnam DF. Sirolimus in blue rubber bleb naevus syndrome: a systematic review. J Paediatr Child Health. (2019) 55:152-5. doi: 10.1111/jpc.14345

55. Ranieri C, Di Tommaso S, Loconte DC, Grossi V, Sanese P, Bagnulo R, et al. In vitro efficacy of ARQ 092, an allosteric AKT inhibitor, on primary fibroblast cells derived from patients with PIK3CA-related overgrowth spectrum (PROS). Neurogenetics. (2018) 19:77-91. doi: 10.1007/s10048-018-0540-1

56. Al-Olabi L, Polubothu S, Dowsett K, Andrews KA, Stadnik P, Joseph AP, et al. Mosaic RAS/MAPK variants cause sporadic vascular malformations which respond to targeted therapy. J Clin Invest. (2018) 128:1496-508. doi: 10.1016/j.jid.2018.03.765

57. Palmieri M, Pinto AM, di Blasio L, Currò A, Monica V, Sarno LD, et al. A pilot study of next generation sequencing-liquid biopsy on cell-free DNA as a novel non-invasive diagnostic tool for KlippelTrenaunay syndrome. Vascular. (2021) 29:85-91. doi: 10.1177/17085381209 36421

58. McNulty SN, Evenson MJ, Corliss MM, Love-Gregory LD, Schroeder MC, Cao Y, et al. Diagnostic utility of next-generation sequencing for disorders of somatic mosaicism: a five-year cumulative cohort. Am J Hum Genet. (2019) 105:734-46. doi: 10.1016/j.ajhg.2019.09.002

Conflict of Interest: The authors declare that the research was conducted in the absence of any commercial or financial relationships that could be construed as a potential conflict of interest.

Publisher's Note: All claims expressed in this article are solely those of the authors and do not necessarily represent those of their affiliated organizations, or those of the publisher, the editors and the reviewers. Any product that may be evaluated in this article, or claim that may be made by its manufacturer, is not guaranteed or endorsed by the publisher.

Copyright (c) 2021 Diociaiuti, Paolantonio, Zama, Alaggio, Carnevale, Conforti, Cesario, Dentici, Buonuomo, Rollo and El Hachem. This is an open-access article distributed under the terms of the Creative Commons Attribution License (CC BY). The use, distribution or reproduction in other forums is permitted, provided the original author(s) and the copyright owner(s) are credited and that the original publication in this journal is cited, in accordance with accepted academic practice. No use, distribution or reproduction is permitted which does not comply with these terms. 